\title{
Three-dimensional dynamic Monte Carlo simulations of elastic actin-like ratchets
}

\author{
N. J. Burroughs, D. Marenduzzo \\ Mathematics Institute, University of Warwick, Coventry CV4 7AL, England
}

\begin{abstract}
We present three-dimensional Monte-Carlo dynamic simulations of the growth of a semiflexible fibre against a fluctuating obstacle. The natural reference for our numerical study are the elastic and brownian ratchet models previously analysed semi-analytically. We find that the decay of the velocity versus applied load is exponential to a good degree of accuracy, provided we include in the load the drag force felt by the moving obstacle. If fibre and obstacle only interact via excluded volume, there are small corrections to the brownian ratchet predictions which suggests that tip fluctuations play a minor role. If on the other hand fibre and obstacle interact via a soft potential, the corrections are much larger when the obstacle diffuses slowly. This means that microscopic assumptions can profoundly affect the dynamics. We also identify and characterise a novel "pushing catastrophe" - which is distinct from usual fibre buckling - in which the growth of the fibre decouples from the obstacle movement. The time distribution of catastrophes can be explained via an approximate analytical treatment, and our numerics suggest that the time taken to lose propulsive force is largely dependent on the fibre incidence angle. Our results are a first step in realising numerical polymer models for the motion of sets or networks of semiflexible fibres close to a fluctuating membrane or obstacle.
\end{abstract}




\section{INTRODUCTION}

Fibre polymerisation or depolymerisation is a common process in eukaryotic cells, which is believed to play a key role in a number of phenomena ranging from cell motility to chromosome separation in mitosis [1-4]. Possibly the clearest example in which polymerisation exerts a force in vivo is represented by the actin-based motility of intracellular viruses and bacteria, such as the well studied Listeria monocytogenes [2]. In the case of Listeria, bundles of actin form "comet tails" which appear to push the bacterium through the cytoplasm of an infected cell. Furthermore, experiments have also been performed to estabilish the minimal numbers of constituents which are necessary to reproduce motility.

A well known model for polymerisation induced motility is the brownian ratchet (BR) model introduced by Peskin et al. in 1993 [5]. According to the BR model, the membrane or bacterium in front of the polymerising fibre, be it actin or microtubule, undergoes thermal diffusive motion. Such a motion is random but can be rectified by the fibre, which is modelled as an advancing rod. This model neglects the fibre elasticity, and as a consequence is amenable to an analytic treatment, which predicts a functional form for the velocity load curves, in good semi-quantitative agreement with experiments [6]. The brownian ratchet model has been generalised to the case in which the fibre is modelled as an elastic fibre [7], which itself undergoes thermal fluctuations. In this version the model is usually referred to as the elastic ratchet (ER). Now the obstacle motion can proceed via two distinct mechanisms: either by rectifying the obstacle diffusion or via bending of the fibre, which allows monomer intercalation, and then straightening of the fibre as the obstacle moves.

The ratchet models have since been refined by many authors, who have considered more comprehensive versions taking into account possible tethering of the fibre to the obstacle [8], a better characterisation of the obstacle as a fluctuating membrane $[9,10]$ and other effects. Typically, these papers tend to concentrate on analytically or semi-analytically treatable models. It would be desirable to study the same system via numerical simulations, which would then clarify the domain of validity of the analytical approximations.

We present three-dimensional Monte Carlo dynamic simulations of the growth of a semiflexible polymer, mimicking an actin or a microtubule fibre, against a fluctuating load modelling a 
bacterium in the cytoplasm or a patch of a membrane. There are only few related numerical "microscopic" simulations we are aware of, the simulation in two dimensions of a single growing elastic fibre [11], and stochastic growth simulations in three dimensions of an infinitely stiff actin branched network [12]. Our simulations are three dimensional, and allow us to statistically study the problem of polymer buckling and of a novel phenomenon which we call a "pushing catastrophe" - when the growth of a polymer becomes decoupled from the obstacle movement. This is not currently appreciated and should be of use when designing more coarse-grained polymer ratchet models which describe fibres with only a few variables. As the algorithm fully takes into account the fibre elasticity, we are able to determine whether the obstacle moves mostly by rectifying (ratcheting) its diffusion or by rectifying the tip fluctuations.

The Monte Carlo local dynamics we use, also known as the "kink-jump" algorithm, has recently been successfully applied to many polymer problems, both on and off lattice [10, 13-20], and naturally fits the stochastic nature of the growth rules typically used in the ratchet literature.

This work is structured as follows. In the next section, we introduce the Monte-Carlo dynamic kink-jump algorithm and we describe how the original algorithm needs to be complemented to be applied to our growth case. Then in section III we discuss our results: first, we present the averaged wall and fibre growth velocity versus load curves; second, we report the time distribution of fibre buckling and 'pushing' catastrophes under a load. At the end of the section we discuss how these results are expected to change as the model is modified in various ways. Section IV contains our conclusions. A few technical details on drag force effects and buckling of obliquely incident fibres are discussed in two Appendices.

\section{SIMULATION MODEL}

We simulate the dynamics of a semiflexible polymer growing against a fluctuating obstacle a two-dimensional wall parallel to the $x y$ plane (Fig. 1) using three-dimensional dynamic Monte Carlo simulations. Our aim is to reconstruct, by averaging over many stochastic realisations of the growth process, (a) the average growth velocity as a function of the loading force, and (b) the temporal statistics of chain buckling (and/or catastrophes, see section III) under a load. Our 
simulation method is similar in many respects to the one recently employed (in different contexts) in $[17,18]$.

An actin fibre is modelled by a (time-dependent) number, $N(t)$, of hard spheres of diameter $h$ (labelled by their three-dimensional space positions $\left\{\vec{r}_{i}\right\}_{i=1, \ldots, N(t)}$ ), which are freely joined together by $N(t)-1$ inextensible links of length $a$. We denote the link joining the $i-$ th and the $(i+1)$-th sphere $\vec{t}_{i}$. For each trial move, we attempt to rotate a bead through a random angle - the bead is chosen randomly from the $N$ constituting the chain - around the axis joining its first nearest neighbours along the chain. The angle is chosen with uniform probability in the $\left[-\theta_{0}, \theta_{0}\right]$ interval. If an end sphere is chosen, we rotate the terminal link around a randomly chosen axis (chosen uniformly from the unit sphere) through a random angle in the $\left[-\theta_{0}, \theta_{0}\right]$ interval. Throughout the simulations $\theta_{0}$ is a constant. If $\theta_{0}$ is small enough this procedure is guaranteed to be equivalent to the generation of a gaussian noise. However we checked that the results were independent of $\theta_{0}$. In particular $\theta_{0}=\pi$ allows a larger time step, and we used this value in the simulations reported below. This choice is commonly made in dynamic studies with the kink-jump algorithm.

There is an interaction energy, $H$, between neighbouring spheres on the chain, which is due to the semiflexible nature of the fibers, and is given by:

$$
H=-K_{b} \sum_{i=1}^{N-2} \vec{t}_{i} \cdot \vec{t}_{i+1}+\sum_{i<j, i, j=1}^{N} V_{\text {h.c. }}\left(\left|\vec{r}_{i}-\vec{r}_{j}\right|\right),
$$

where $K_{b}>0$ is called the bending rigidity and $V_{\text {h.c. }}$ is the hard core potential, which disallows configurations with any two sphere centres closer than $h$ (giving them an infinite weight). If $K_{b} \gg 1$, the persistence length of the fibers, $\xi$, is $\sim K_{b} a$. This Hamiltonian determines the dynamics as after each trial move we compute the change in energy $\Delta H$ and accept the move with probability:

$$
W=\min \{\exp (-\beta \Delta H), 1\}
$$

where $\beta=1 /\left(k_{B} T\right), k_{B}$ being the Boltmann constant which in our units is set to 1 . We note that this scheme ensures that no chain intersection occurs during any attempted rotation of the links provided that the ratio between hard core diameter and bond length is large enough, precisely one needs $h / a>(3 / 4)^{1 / 2} \sim 0.866$. We always chose $h / a=0.9$ in the simulations reported below unless specified otherwise. 
The obstacle is fluctuating and undergoes a random walk in time. We do this by choosing with uniform probability a trial new position within a distance $\delta l$ (along the $z$ axis, see Fig. 1) from the old one. If no load is applied then the obstacle simply undergoes brownian motion, and it can be shown that the diffusion constant of the obstacle is $D_{o}=(\delta l)^{2} / 6$. In all cases we reject the trial position if any of the beads making up the fibre overlaps with the obstacle.

The semiflexible fibre is growing at one end. We model this by proposing an additional "move" in our three-dimensional Monte Carlo dynamics: with probability $k_{\text {on }}$ we try to add one monomer to the end of the fibre, i.e. attached to the monomer in $\vec{r}_{\mathrm{N}(\mathrm{t})}$. The direction of the new link $\vec{t}_{\mathrm{N}(\mathrm{t})}$ is chosen according to the probability distribution:

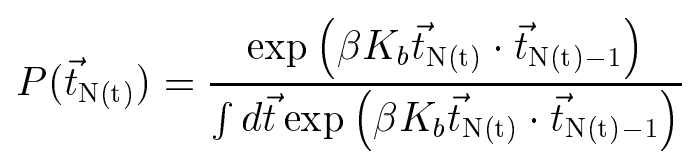

If the proposed new link crosses the obstacle position we reject the growth move. As the bending rigidity $K_{b}$ is always very large in the cases considered here, this procedure is basically equivalent to choosing the new link in the direction of the last link of the already grown polymer, $\vec{t}_{\mathrm{N}(\mathrm{t})-1}$.

We consider clamped boundary conditions for the fibre at the other end-i.e. we assume that both the first bead and the first link (the local tangent there) are fixed. The wall is initially at a (time-dependent) position $z(t)$, positioned randomly at a distance of within 2 beads from the fibre tip, and the first link $\vec{t}_{1}$ is taken to lie in the $x z$ plane, making an angle $\theta$ with respect to the $z$ axis. Results presented here are unaffected by the exact initial conditions chosen.

Fig. 1 shows the set-up considered in the simulations used throughout. The parameters of the models are: the growth rate $k_{\text {on }}$ at the free end (typically 0.001 in simulation units); the angle $\theta$; the persistence length $\xi$ of the fiber (typically one to several $\mu \mathrm{m}$ for actin); the diffusion coefficient of the obstacle $D_{o}$, and that of the fibre tip, $D_{t}$; and finally the load on the obstacle, which we introduce as a downward pushing force $f$.

Time is measured in Monte-Carlo sweeps. A Monte-Carlo sweep is defined as a sequence in which, on average, all the beads in the polymer and the obstacle have had the possibility to move once [14]. Thus as the fibre grows this unit step involves more elementary computations (trial moves). It is useful here to set the mapping between real and simulation units used throughout this work (naturally as ours is a Monte-Carlo dynamics exact values coming from this mapping 
will have to be treated cautiously). One bond length $a$ is set equal to $5 \mathrm{~nm}$, which is the diameter of an actin fibre. To set the time scale, we identify the value $D_{o}=0.00167$ with a target value in physical units, e.g. $D_{o}^{\text {real }} \sim 10^{-2} \mu \mathrm{m}^{2} \mathrm{~s}^{-1}$ (intermediate between a fluctuating membrane and a fluctuating bacterium [7] and such that the fibre can in principle move at the polymerisation speed). A Monte-Carlo sweep then corresponds to $\Delta t \sim a^{2} D_{o} / D_{o}^{\text {real }}$, which with the choices above is $\Delta t \sim 4.2 \mu \mathrm{s}$. The numbers in simulation units given in the results can then be converted into real units accordingly. For example a persistence length of $K_{b} a=2000$ corresponds to 10 $\mu \mathrm{m}$, and a $k_{\mathrm{on}}=10^{-3}$ to a polymerisation rate of $\sim 240 \mathrm{~s}^{-1}$. The fluctuations of the tip can be quantified in a similar way. In the case of a flexible chain $\left(K_{b}=0\right)$ the tip angular diffusion is then $\theta_{0}^{2} /(6 \Delta t)$ (measured in $\left.\operatorname{rad}^{2} s^{-1}\right)$. With the values we chose this corresponds to $D_{t} \sim 10^{5} \operatorname{rad}^{2} s^{-1}$ (for $K_{b}=0$ ). The bending rigidity renders the tip diffusion slower than this "bare" value.

\section{RESULTS}

\section{A Velocity laws}

In this section we estimate the velocities of the wall and the growth velocity of the tip as a function of the load impinging on the obstacle.

It is useful first to summarise theoretical expectations based on semi-analytical treatments within the brownian ratchet $(\mathrm{BR})$ and elastic ratchet $(\mathrm{ER})$ models $[5,7]$. In the BR models, the fibre is infinitely stiff and movement occurs when the thermal diffusion of the obstacle allows for a gap of size $a$ to appear. In this case Peskin et al. [5] have shown that if the diffusion of the obstacle is very fast with respect to the polymerisation kinetics, i.e. if $\frac{D_{o}}{k_{o n} a^{2}} \gg 1$, then the growth rate of the fibre $v_{g}$ (measured in monomer per second) and the wall velocity $v$ are respectively given by (in the absence of depolymerisation):

$$
\begin{aligned}
v_{g} & =k_{o n} \exp \left(-\frac{f a \cos (\theta)}{k_{B} T}\right) \\
v & =v_{g} a \cos (\theta)
\end{aligned}
$$

where $\theta$ is the angle between the filament and the wall normal (see Fig. 1). 
In the ER model, the hypothesis that the filament is infinitely stiff is relaxed. The idea is that even if the obstacle diffusion is quite slow, Eq. 4 still holds because the filament tip fluctuations compensate and it is those fluctuations which can be rectified by the polymerisation kinetics. As a result, the same equations hold to a good approximation (at least for a polymer which is not too flexible, see Ref. [7]).

The average wall velocity at zero load and normal incidence in the BR is bounded by [5]:

$$
v_{\max , \mathrm{BR}}=\frac{2 D_{o}}{a}
$$

which is known as the velocity of the ideal BR. This velocity, when the fluctuations of the obstacle are taken to correspond to the motion of the whole bacterium, may be too small to account for the experimentally observed velocities in bacteria such as Listeria, hence the rationale for introducing the ER model. Another obvious bound on the growth velocity, holding for both the BR and the ER models, on the other hand, is given by $k_{o n} a$.

We now turn to our numerical results for the polymer ratchet in three dimensions. Fig. 2a shows an example of averaged obstacle position versus time, and also displays two typical runs. Fig. 2b shows how this average varies with the applied load (see Fig. 1). From the figure we see that a linear region for early times can be identified in all curves. Therefore from the slope of these curves we could determine the wall velocities. Fibre growth velocities were determined by an analogous procedure.

Fig. 3a shows the velocity - force law for three fibers, (persistence length $10 \mu \mathrm{m}$, assuming $a=5 \mathrm{~nm}$, see section II), moving against obstacles with different $D_{o}$ (i.e. different mobilities), in the $(f, \log v)$ plane. The incidence is normal, i.e. $\theta=0$. The data suggest an approximately exponential decay with a decay constant dependent on the force, and increasing with increasing force. For large load, the curve approaches the theoretical prediction $v \sim \exp \left(-\frac{f a}{k_{B} T}\right)$ which can be obtained on the basis of the kinetic models considered in Refs. [5, 7, 8], but for smaller load the data show a strong deviation.

This discrepancy is reduced and the decay is approximately exponential if the velocity data are plotted against the quantity $f_{\text {tot }}=f+\Gamma v$, which is the load force plus the drag force $\Gamma v$, where $\Gamma=k_{B} T / D_{o}$ (Fig. 3b). Then the data with large diffusion coefficients are in very good agreement 
with the analytical predictions of the BR and ER models valid for fast diffusion of either the tip or the obstacle. However, if $D_{o}$ becomes small, the data suggest a law $v=A \exp \left(-f_{\text {tot }}\right)$ to hold, with $0<A<1$ a prefactor which depends on $D_{o}$.

We also performed simulations with more flexible fibres, persistence lengths of 1 and $2.5 \mu \mathrm{m}$, obtaining the same trend. It is to be noted that the velocity versus load curves are computed by considering only fibers which have not yet undergone a "catastrophe" (see below for a definition of fibre catastrophe in our simulations).

Fig. 4 shows the effect of changing the incidence angle, and compares the trends of wall and growing velocities. Broadly, the results are qualitatively equivalent as the ones obtained for normal incidence. However, for the same value of $D_{o}$, the correction to the ideal exponential decay in Eq. (4) is smaller for fibres incident at an angle. For large $f$, and $\theta=\pi / 4$, the simulated fibre growth velocity (Fig. 4b) slightly exceeds the theoretical prediction from the ratchet analytic models (Eq. (4)). Since in Fig. 4a we observe no such effect in the wall velocity curve, we conclude that this is due to a bending of the fibre at the tip, which allows a faster growth as the gap required for monomer intercalation is smaller. Contrarily, the data corresponding to the $\pi / 6$ wall velocities (Fig. 4c) show some evidence of a slightly larger slope (in the linear logarithmic plot) than the one predicted by the ratchet models, which may result from the fact that $\pi / 6$ is a non-optimal angle and bending away from it can enhance the obstacle speed.

It is interesting to ask whether in our simulations the velocities observed are mainly due to rectifying the fluctuations of the obstacle as in the BR model or those of the filament tip as in the ER model, as both mechanisms are in principle viable and incorporated in our simulations. From Figs. 3 and 4 it is already clear that the tip fluctuations cannot fully compensate in the case of slow obstacle diffusion, so that the velocity versus force curves fall below the ideal ratchet curves - in other words there is a large portion of simulated points for which the velocity is limited by $D_{o}$. A simple criterion to quantify the contribution of the tip fluctuations is to compare the obstacle velocities to the predictions coming from the BR model, the implicit solution of which is reported in Ref. [5]. As the BR model considers an infinitely stiff polymer, the predicted velocity is simply due to rectifying the obstacle thermal diffusion, and thus it will be smaller than the one observed in our simulations. The extra velocity we observe is then entirely due to the tip fluctuations. 
Fig. 5 shows the obstacle velocity as a function of $D_{o}$ for (a) $\theta=0$ and (b) $\pi / 4$, and zero applied load, and compares this with the velocity predicted in the BR model with the same parameters. We see little evidence of a deviation from the BR behaviour even for very small diffusion coefficients (down to $D_{o}=1.5 \times 10^{-6}$ for normal incidence and $D_{o}=1.67 \times 10^{-7}$ for $\theta=\pi / 4$ ). The obliquely incident fibres display a larger correction coming from the tip fluctuations. This should qualitatively be expected as it was proved in Ref. [7] that the system is equivalent to a "spring" whose constant, for $\theta$ not too small, is proportional to $\sin \theta^{-2}$. However, an analytical quantitative estimate for the correction to the BR model due to tip fluctuation is not presently available. The full numerical simulations presented here seem to be the cleanest way to quantify this correction as they do not involve any other approximations apart from those defining the ER model.

We can also monitor the deviation from the BR model in the full velocity versus load curve, i.e. not only for zero load (see Fig. 6, (a)-(c)). We find that for $k_{\mathrm{on}}=10^{-3}$ and $D_{o}=10^{-5}$, the velocity of the obstacle is $\sim 40 \%(\theta=0, \pi / 6)$ and $\sim 50 \%(\theta=\pi / 4)$ higher than the BR prediction. Thus for oblique incidence we have a larger absolute deviation.

Consequently, we conclude that within the phase space covered by our simulations the order of magnitude of the wall velocity is governed by the rectification of the obstacle diffusion, and the role of tip flexibility is minor in determining the $v-f$ relation.

\section{B Statistics of fibre buckling and of pushing catastrophes}

As our simulations include the polymer elasticity, we observe that at certain times the fibre bends and typically starts to grow in the plane of the obstacle or away from it. This happens (a) either when it is growing under too large a load (more precisely load plus drag force) (b) or when it has grown too long. When a fibre starts to bend over it is typically very difficult for it to recover to the straight configuration (favoured by the bending rigidity potential) since unhindered polymerisation rapidly extends the fiber. In other words, the fibre growth and the obstacle movement have now become uncorrelated. We call this phenomenon a "pushing catastrophe". Fig. 7a shows an example of configuration of a fibre which has undergone a "pushing catastrophe". Our aim in this section is to characterise the probability distribution of the times at which such catastrophes 
occur.

We stress that the pushing catastrophe we identify is quite distinct from the classic Euler buckling in which the fibre deviates from the straight conformation - favoured by the its stiffness - in the presence of a load. If the fibre is normally incident to the wall there is a well defined buckling transition such that if the force exceeds a length dependent threshold the fibre bends. However, for obliquely incident fibres this threshold is zero so that the phenomenon of buckling as a phase transition is not present in this more general case (see Appendix B).

Nevertheless, it still makes sense to consider events leading to the decoupling between growth and movement. These are precisely the "pushing catastrophes" or catastrophes. It is thus useful for our purposes to have an operational definition of a catastrophe. In Fig. 7b the times corresponding to a catastrophe can be identified as those at which the trajectories in the $(z(t), N(t))$ plane (the two variables denote respectively the instantaneous wall position and fibre length at time $t$ ) diverge from the ideal curve corresponding to the case in which growth is coupled to wall movement (as a threshold in our simulations we typically required that the $\frac{z(t)-N(t) \cos (\theta)}{N(t) \cos (\theta)}>0.3$ if $\theta$ is the fibre initial incidence angle, see Fig. 1).

Fig. 8a shows the distribution of catastrophe times for a fibre with persistence length $2.5 \mu \mathrm{m}$ and normally impinging on the fluctuating obstacle. It can be seen that as the load on the obstacle increases, the mean of the catastrophe time distribution increases and the distribution broadens. It is possible to understand in a qualitative way this behaviour by a simple argument. Let us restrict to the case of normal incidence for concreteness. At least if the force is large enough, once the fibre undergoes a catastrophe there can be no recovery. The average time needed for the system to undergo a pushing catastrophe is:

$$
\tau_{\text {cat }} \sim \exp (\Delta E)
$$

where $\Delta E$ is the energy lost by fibre bending. We can estimate $\Delta E$ by using Eq. 13 (see Appendix B) with a radius of curvature $R \sim L$. We then obtain $\Delta E=-A f L+B / L$, where $A$ and $B$ are constants, $f$ is the load force, and $L \equiv L(t)=N(t) a$.

Let now $q(t)$ be the fraction of the fibre population which has not undergone a catastrophe by time $t$. If we assume for simplicity that $q(t)$ obeys a simple relaxation dynamics at all lengths (in the quasi-static approximation), Eq. 6 leads us to consider the following simplified dynamic 
equation for $q(t)$ :

$$
\frac{d q(t)}{d t}=-\frac{C(t)}{\tau_{\text {cat }}} q(t)
$$

where $C(t) \equiv C(L(t))$ is a coefficient characterising the configuration relaxation, which depends on the fibre length at time $t, L(t)$. In order to make contact with the numerics we need to assume that $C$ depends on $L(t)^{2}$ (see caption to Fig. 8).

From the results reported in the previous section for the fibre growth velocity we expect $L(t) \sim$ $r t \exp (-\beta f)$, with $r>0$ a constant, so that the probability $p_{\text {cat }}(t)$ that the fibre undergoes a pushing catastrophe at time $t$ is:

$$
p_{\text {cat }}(t)=-\frac{d q(t)}{d t} \propto C(t) \exp (-\Delta E(t)) \exp \left(-\int_{0}^{t} d t^{\prime} C\left(t^{\prime}\right) e^{-\Delta E\left(t^{\prime}\right)}\right)
$$

In Fig. 8b we illustrate this prediction, fitting the constants introduced above. There is a good qualitative agreement with the simulation data, even if it is difficult to guess a priori the exact value of the constants involved in the approximate analytical treatment.

Fig. 9 shows the effect of changing the incidence angle. As shown in Appendix B, when the fiber is not normally incident to the wall it buckles under even an infinitesimally small load. However, catastrophes are still well defined in our simulations. Our data suggest that as the angle between fiber and wall normal increases (cases of $\pi / 6$ and $\pi / 4$ incidence are shown in Fig. 9a and $9 \mathrm{~b}$ respectively), the difference between the time distribution of catastrophes becomes progressively smaller.

\section{Modifying model assumptions}

The results presented so far were obtained with a minimal model for the growth dynamics of a semiflexible polymer near a diffusing wall. All interactions except for the bending rigidity of the fibre are imposed via excluded volume effects. It is interesting to ask what happens when the assumptions underlying the model are modified to critically assess the consequences of all our assumptions in section II.

First, let us consider the case of a soft potential between fibre and obstacle. Instead of simply excluding the configurations where the fibre crosses the obstacle, we penalise them with a potential 
( $a_{1}$ and $a_{2}$ are two positive constants):

$$
V_{\mathrm{soft}}=\sum_{i=1}^{N(t)} a_{1}\left|z_{\mathrm{wall}}-z_{i}\right|^{a_{2}} \Theta\left(z_{i}-z_{\mathrm{wall}}\right)
$$

with $\Theta$ denoting the Heaviside step function, and where $z_{\text {wall }}$ and $z_{i}$ are the position along the $z$ axis (Fig. 1) of the obstacle and the $i$-th bead respectively. We considered a first case, which corresponds to a very soft wall, in which $\left(a_{1}, a_{2}\right)$ were taken to be equal to $(1,2)$ (very hard wall), and another one in which they were equal to $(10,4)$, which corresponds to a very hard wall. Fig. 10a shows the comparison between the very soft wall and the excluded volume wall considered before for $D_{o}=0.00015$. The very soft wall moves only slightly faster. Fig. 10b compares very soft, very hard and excluded volume walls with a smaller $D_{o}$, and clearly shows that if $D_{o}$ is small, then the wall moves faster as it becomes softer. Indeed from Fig. 10b it can be seen that the very soft wall can significantly overcome the ideal BR limit.

We note that when the wall becomes "soft", we also need to modify the rule by which we grow the fibre. In our simulations we have assumed that if the proposed position for the newly grown bead (the $N(t)$-th) is within the obstacle, we accept this move with probability $\exp (-\epsilon)$, where:

$$
\epsilon=\frac{a_{1}\left|z_{\mathrm{wall}}-z_{N(t)}\right|^{a_{2}}}{k_{B} T}
$$

Other rules may lead to different wall and fibre growth velocities in principle.

We also performed simulations without imposing self-avoidance, i.e. with $h / a=0$. It has to be noted that the simulations performed with the kink-jump algorithm only make sense if $h / a=$ 0 or $h / a>\sqrt{3 / 4}$, otherwise transient violation of self-avoidance can occur during the local crankshaft or pivot moves (see section II). In the parameter region explored here, with $K>500$ and $N(t)<40$, self-avoidance does not appreciably affect the velocity versus load curves. As the fibre gets floppier or longer, we expect that kinking can occur, which should eventually affect the time distribution of catastrophes.

Another ingredient which may be of relevance in real actin and microtubule fibres are the intrinsic twist and twisting or torsional rigidity. Again we expect this not to change the velocity versus load curves qualitatively, while the buckling and catastrophe statistics may change with respect to those valid for the semiflexible fibre treated here. 


\section{DISCUSSION AND CONCLUSIONS}

In this work we have presented three dimensional Monte Carlo dynamic numerical simulations of the growth of a semiflexible polymer against a fluctuating obstacle with an applied load. We computed the average wall and tip growth velocity as a function of the applied load and compared to analytical predictions. We found that the velocity decays roughly exponentially with the load (as predicted by the elastic and brownian ratchet models for a fast diffusing obstacle or tip, but with a coefficient depending on the obstacle diffusion constant) provided that the drag force acting on the obstacle is taken into account. An analysis of the analytical solution of the brownian ratchet model in Ref. [5] shows why this correction is needed (see Appendix A). Our simulations also allow us to quantitatively measure the contribution of tip fluctuations to the obstacle velocity. Within the parameter range explored here, the maximal correction to the brownian ratchet prediction is $50 \%$ for $45^{\circ}$ fibre incidence and very slow obstacle diffusion. This suggests that the main movement mechanism - at least in the parameter space explored in our simulations - is the one in which the fibre growth rectifies the obstacle thermal diffusion and the tip fluctuations do not play a major role in determining the obstacle velocity.

Further, our calculations show that at all angles of incidence the fibres at a certain stage of growth bend and start to grow parallel to the plane of the obstacle or even away from the obstacle. We call this phenomenon a pushing catastrophe, a process distinct from classical buckling, which is an equilibrium phenomenon. The time distribution of such catastrophes for normal incidence tends to broaden and its average tends to increase, as the load increases. These findings are in qualitative agreement with a simplified semi-ananlytical treatment. As the initial angle between the fibre tip and the wall normal increases, we find catastrophes happen earlier and their time distribution is less sensitive to the applied load.

We also show that microscopic assumptions in the model can profoundly affect the growth dynamics, so that as the interactions between fibre and obstacle becomes softer - i.e. if we relax the assumption of excluded volume interaction between fibre and obstacle - then the obstacle moves faster when all other parameters are the same.

We anticipate that our results will be useful in designing more complicated polymer models for 
cell motility and cytoskeleton dynamics, where the full network of semiflexible and polymerising fibre is considered instead of a single fibre. As it is very difficult with present day computing power to model a large enough number of filaments to mimick the cytoskeleton of an eukaryotic cells, it may prove necessary to coarse grain the filaments further, e.g. considering only a limited number of bending modes. The rules determining these coarse graining and the evolution of the network might then be chosen according to the full numerical solution for a single three dimensional fibre presented here.

Acknowledgements: This work was supported by EPSRC grant GR/S29256/01.

\section{APPENDIX A: THE BROWNIAN RATCHET FOR VERY FAST OBSTACLE DIFFUSION}

In this Appendix we work out a simple limit of the ratchet model which illustrates how the drag force correction highlighted in the simulation results appears.

Let us consider the brownian ratchet model, in which the polymer is infinitely stiff and the obstacle is diffusing with diffusion constant $D_{o}$. If $D_{o}$ is infinite, then the growth is unobstructed and the fibre growth velocity is equal to the polymerisation velocity. In Ref. [5] it was shown that for a generic value of $D_{o}$ the wall velocity is (in the absence of a load, i.e. take the limit as $\omega \rightarrow 0$ of the velocity vs load curve, Eq. (2) of Ref. [5]):

$$
v=\frac{2 D_{o}}{a} \frac{\mu}{1+\mu / 2}
$$

where $\mu$ is given by the solution of the equation $\mu=\frac{k_{o n} a^{2}}{D_{o}} \frac{1-\exp (-\mu)}{\mu}$. Let us expand $\mu$ and consequently $v$ in powers of $1 / D_{o}$ keeping only the terms linear in $1 / D_{o}$. We find that:

$$
\begin{aligned}
\mu & \simeq \frac{\frac{k_{o n} a^{2}}{D_{o}}}{1+\frac{k_{o n} a^{2}}{D_{o}}} \\
v & \simeq k_{o n} a\left(1-\frac{k_{o n} a^{2}}{D_{o}}+O\left(1 / D_{o}^{2}\right)\right) \\
& \simeq k_{o n} a\left(1-\beta f_{\text {drag }} a\right) \\
& \simeq k_{o n} a \exp \left(-\beta f_{\text {drag }} a\right)
\end{aligned}
$$

where we have put in the definition of drag force, i.e. $f_{\text {drag }}=\beta v / D\left(\beta=\frac{1}{k_{B} T}\right)$. Eq. 12 shows that even at large diffusion and in the absence of an applied load the tip cannot advance at the 
polymerisation speed, but one has an exponential decay in which the force includes the drag force contribution.

\section{APPENDIX B: BUCKLING INSTABILITY OF OBLIQUELY AND NORMALLY INCIDENT FI- BRES}

In this Appendix we show that obliquely incident fibres are linearly unstable to buckling under an applied load while a normally incident fibre on the other hand undergoes a well defined buckling transition at a finite value of the applied load (plus drag force).

Let us consider a stiff fibre of length $L=N a$ (i.e. we freeze the configuration at a given time during the growth) impinging on the load at an angle $0<\theta<\pi / 2$ on the obstacle (see Fig. 11, our geometrical analysis follows the one of Ref. [7]). There is a load $f$ acting on the diffusing obstacle. Under the action of a load the energy difference between the straight (Fig. 11, left) and the buckled one (Fig. 11, right) is given by:

$$
\Delta E=-f \delta L+\frac{k_{B} T K_{b} L}{2 R^{2}}
$$

where we have considered that the buckled configuration is uniformly bent so that its local radius of curvature is $R$ everywhere, and $\delta L$ is the change in the projection of the end-to-end distance of the filament on to the force direction. Elementary geometrical considerations then lead to:

$$
\Delta E=-f(L \cos (\theta)-R \sin (\theta+L / R)+R \sin (\theta))+\frac{k_{B} T K_{b} L}{2 R^{2}} .
$$

Considering now the onset of buckling, we take $R \rightarrow \infty$ and expand Eq. 14 in powers of $1 / R$, keeping terms up to $1 / R^{2}$. We then get back to the result of Ref. [7] for $\delta L$, which implies:

$$
\Delta E=-f L\left(\cos (\theta) / 6(L / R)^{2}+\sin (\theta) / 2(L / R)\right)+\frac{k_{B} T K_{b} L}{2 R^{2}} .
$$

From Eq. 15 it is apparent that: (a) if $\theta \neq 0$ (oblique incidence), the straight configuration is unstable to buckling for every value of $f>0$, while (b) if $\theta=0$ for $f \rightarrow 0$ the straight configuration is stable, but there exists a threshold $f_{c}$ above which the fibre buckles. The prediction obtained via Eq. 15 for $f_{c}$ is only approximate as we assumed $R \gg 1$. 


\section{REFERENCES}

[1] B. Alberts et al., Molecular biology of the cell, 4th edition, Garland Science (2003).

[2] D. Bray, Cell movements: from molecules to motility, 2nd edition, Garland Publishing (2000).

[3] T. Stossel, Science 260, 1086 (1993).

[4] M. I. Molodtsov et al., P. Natl. Acad. Sci. USA 102, 4353 (2005).

[5] C. S. Peskin, G. M. Odell, G. F. Oster, Biophys. J. 65, 316 (1993).

[6] M. E. Janson, M. Dogterom, Phys. Rev. Lett. 92, 248101 (2004).

[7] A. Mogilner, G. Oster, Biophys. J. 71, 3030 (1996).

[8] A. Mogilner, G. Oster, Biophys. J. 84, 1591 (2003).

[9] D. R. Daniels, M. S. Turner, J. Chem. Phys. 121, 7401 (2004).

[10] S. V. M. Satyanarayana, A. Baumgaertner, J. Chem. Phys. 121, 4255 (2004).

[11] A. E. Carlsson, Phys. Rev. E 62, 7082 (2000).

[12] A. E. Carlsson, Biophys. J. 81, 1907 (2001).

[13] A. Baumgartner, K. Binder, J. Chem. Phys. 71, 2541 (1979).

[14] A. Baumgartner, J. Chem. Phys. 72, 871 (1980).

[15] A. Baumgartner, Annu. Rev. Phys. Chem. 35, 419 (1984).

[16] M. Muthukumar, A. Baumgartner, Macromolecules 22, 1937 (1989).

[17] M. Muthukumar, Phys. Rev. Lett. 86, 3188 (2001).

[18] S.-S. Chern, A. E. Cardenas, R. D. Coalson, J. Chem. Phys. 115, 7772 (2001).

[19] Y. Kantor, M. Kardar, Phys. Rev. E 69, 021806 (2004).

[20] D. Marenduzzo et al., Phys. Rev. Lett. 88, 028102 (2002). 


\section{Figure Captions}

Figure 1: Geometrical set-up used for the three-dimensional dynamic Monte-Carlo simulations. Symbols are defined in the text.

Figure 2: (a) Average over 20 simulations of the wall position as a function of time (in simulation units) for $D_{o}=1.67 \times 10^{-3}$ and normal incidence. We also show two typical growth simulations used for the averaging. Data were collected with a load of $f a / k_{B} T=4, K_{b}=2000$ (10 $\mu \mathrm{m})$. (b) Average wall position as a function of time for three different loads (see legends, in units of $k_{B} T / a$ ). The average slope of the curves is well defined (does not depend on the current length before buckling or a catastrophe - see section III B - occurs) and yields the load-dependent wall velocity via a linear fit.

Figure 3: (Left) Wall velocity versus load force law (in linear-logarithmic plot) for a fibers of stiffness $K_{b}=2000$ (10 $\mu \mathrm{m}$ in our units) and for obstacle of different diffusion constants (in simulation units in the legend). (Right) Same plot but correcting the load force by the drag term. The incidence angle is $\theta=0$ (normal incidence). Each point was obtained by fitting the data as in Fig. 2 and averaging over 20 simulations. This allows an accuracy slightly better than $10 \%$ on the simulation results.

Figure 4: Plots of the wall ((a) and (c)) and fibre growth ((b) and (d)) velocities versus force curves for a fibre at an incidence angle of $\theta=\pi / 4$ ((a) and (b)) and of $\theta=\pi / 6$ ((c) and (d)), for different values of $D_{o}$. The fibre stiffness was $K_{b}=2000$ (corresponding to a persistence length of $10 \mu \mathrm{m})$. Accuracy is between 5 and $10 \%$ on the curves with $D_{o}=0.00167$ and $D_{o}=0.00015$, and between 10 and $20 \%$ on the curve with $D_{o}=0.00001$. Each point on the plot has been obtained by averaging over 20 growth simulations, except for those on the curves for and $D_{o}=10^{-5}$, for which 120 growth simulations were averaged.

Figure 5: Velocity versus $D_{o}$ in $\log -\log$ plot, for zero load, $k_{\text {on }}$ as shown, and $\theta=0$ in (a) and $\theta=\pi / 4$ in (b), with stiffness $K_{b}=2000$. The lines are predictions coming from the 
numerical solution of the BR equations in Ref. [5] (see legends for the exact values of parameters).

Figure 6: Comparison between the velocity versus load curves obtained with the 3-dimensional Monte Carlo dynamics and the predictions from the BR model. Parameters are $D_{o}=10^{-5}$, $k_{\mathrm{on}}=0.001, K_{b}=2000$, while the incidence angle is $\theta=0$ in (a), $\theta=\pi / 6$ in (b) and $\theta=\pi / 4$ in (c). Accuracy on data points is $5 \%$.

Figure 7: (Left) Example of a fibre which has undergone a pushing catastrophe in our simulations. (Right) Two trajectories in the $(z(t), N(t))$ plane, where $z(t)$ and $N(t)$ are the wall position and the fibre length in monomers at time $t$, respectively.

Figure 8: (a) Time distribution probabilities of catastrophes for a fibre with stiffness $2.5 \mu \mathrm{m}$ under different loads found by averaging 90 different simulations with $\theta=0$. We used $D_{o}=1.67$ $10^{-5}$. (b) An example of prediction coming from the approximate treatment in the text (with $A=0.01, B=18.5, r=2.210^{-4}$, and assuming that $C(t)$ is proportional to $L(t)^{-2}$ as for a standard polymer relaxation in the Rouse model).

Figure 9: Time distribution probabilities of catastrophes for a fibre with stiffness $2.5 \mu \mathrm{m}$ under different loads found by averaging 90 different simulations with incidence $\theta=\pi / 6$ (a) and $\theta=\pi / 4(\mathrm{~b})$.

Figure 10: Wall position versus time curves for (a) $D_{o}=0.00015$ (a) and (b) $D_{o}=0.0000015$. In (a) we compare the excluded volume and the very soft wall case. In (b) curves from bottom to top refer to excluded volume, very hard and very soft wall respectively. The fibre incidence is normal and the fibre stiffness is $K_{b}=2000$ in these graphs.

Figure 11: Geometry for Appendix B. A straight (left) and a buckled (right) fibre are shown. Symbols are defined in the text. 


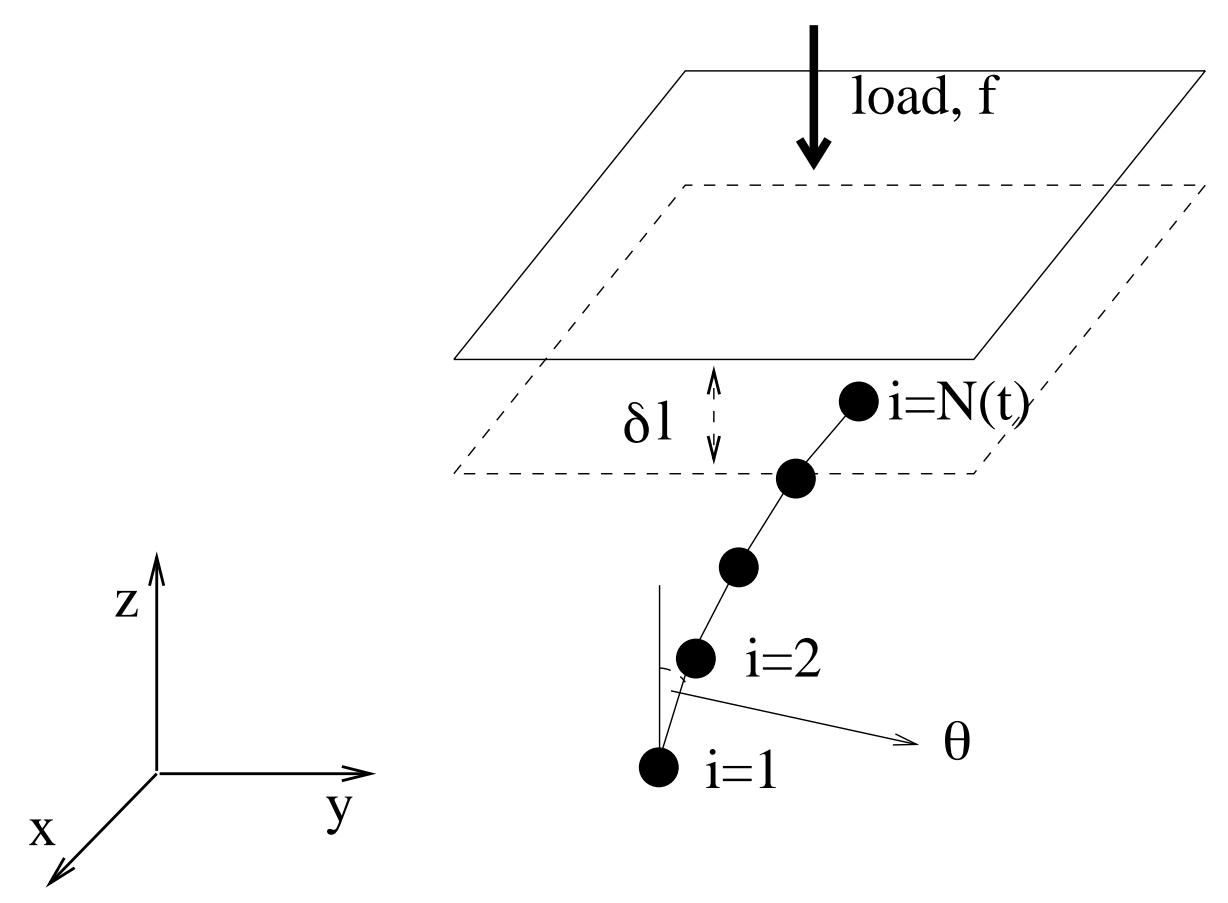

FIG. 1: 

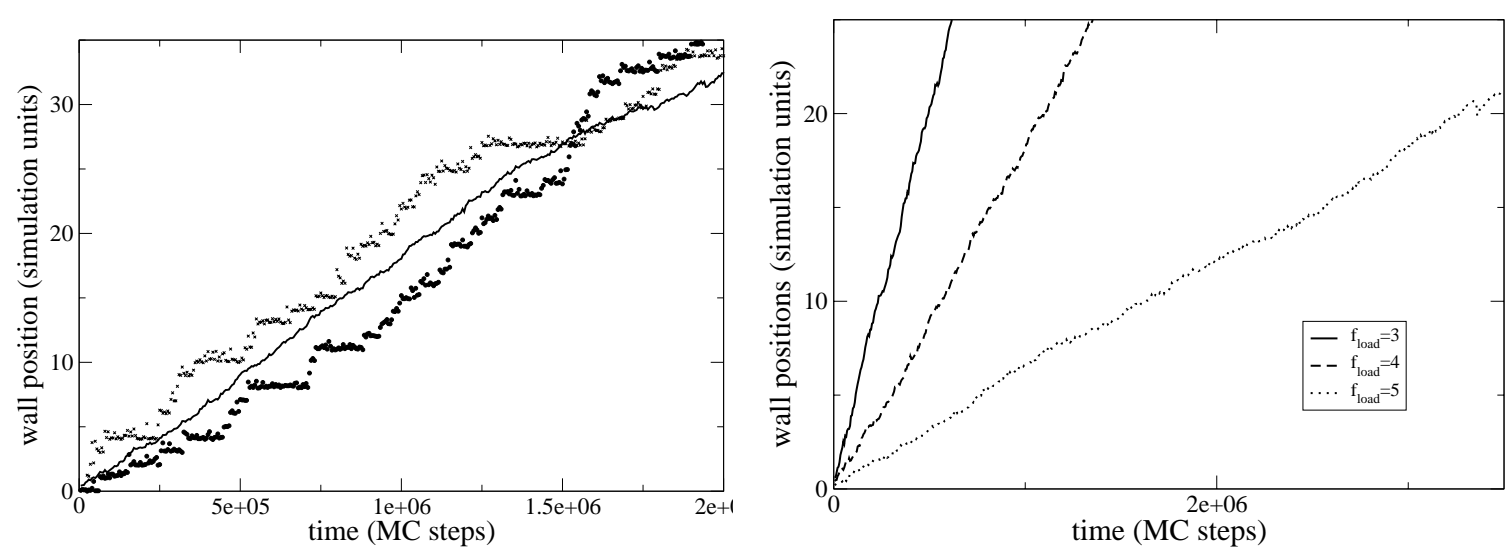

FIG. 2:

(a)

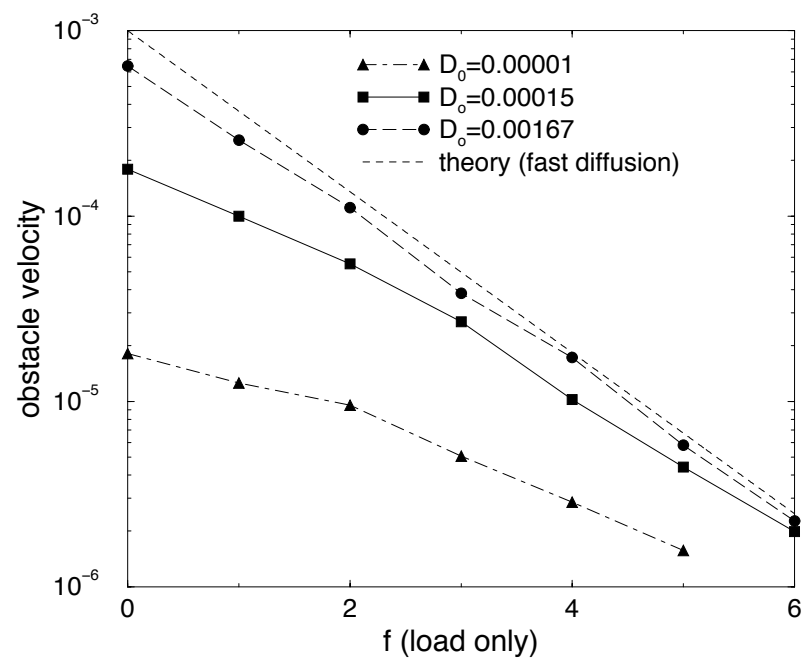

(b)

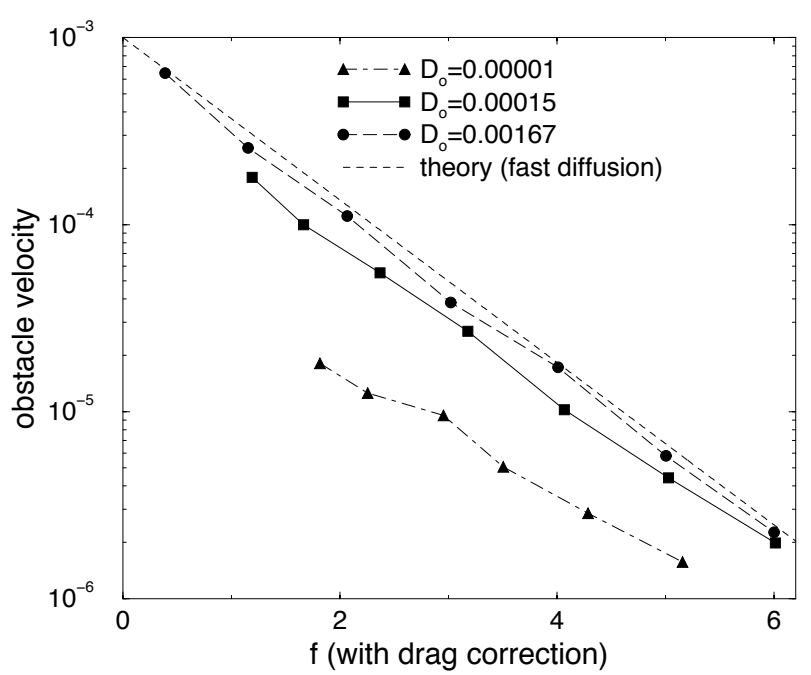

FIG. 3: 
(a)

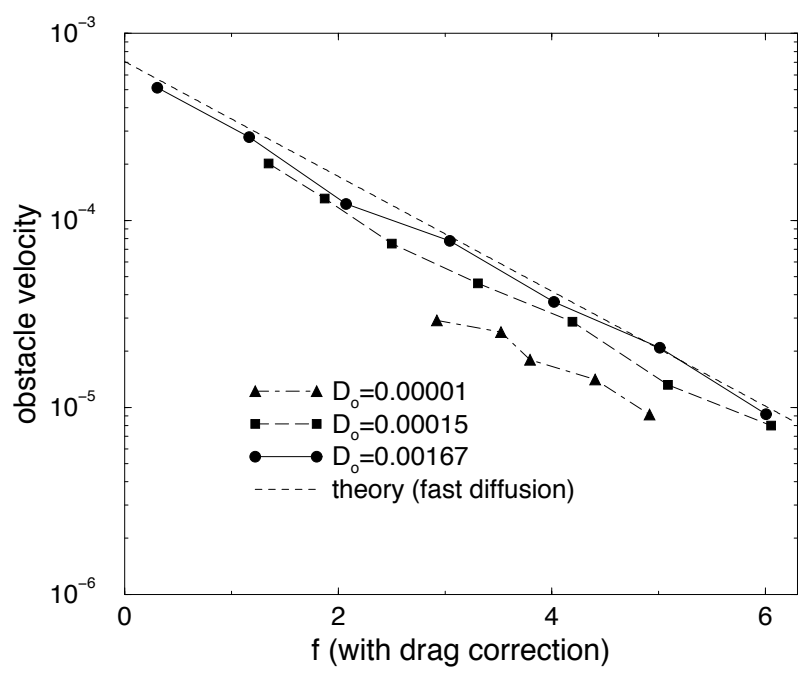

(c)

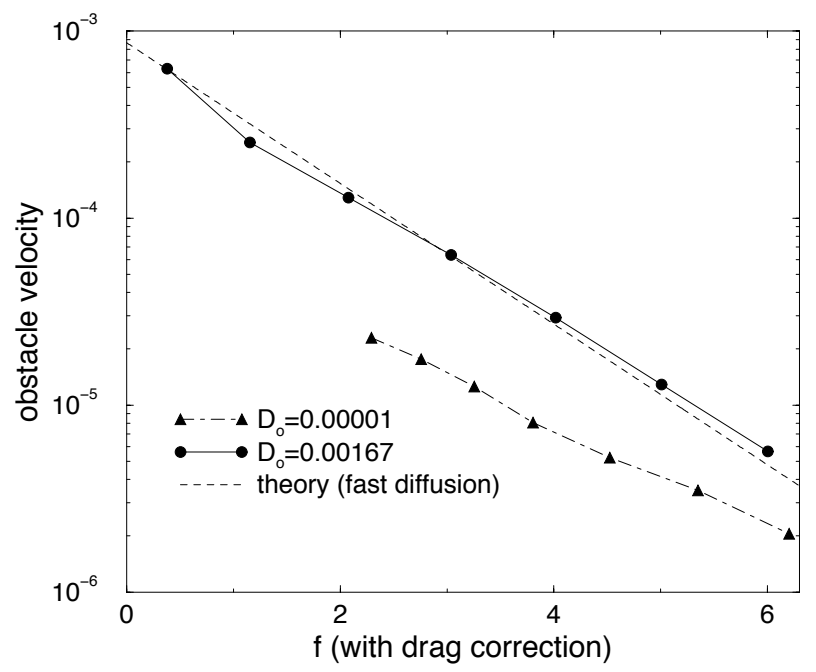

(b)

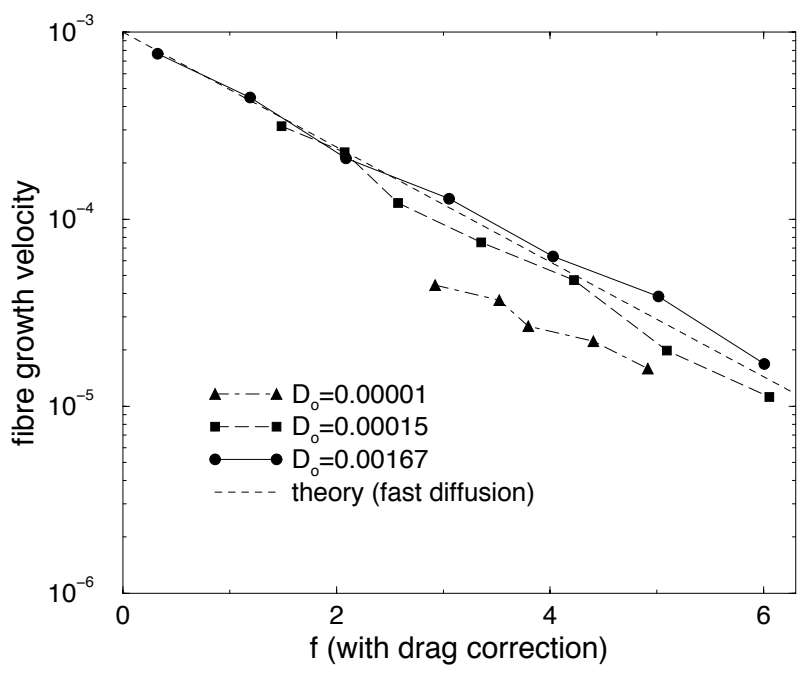

(d)

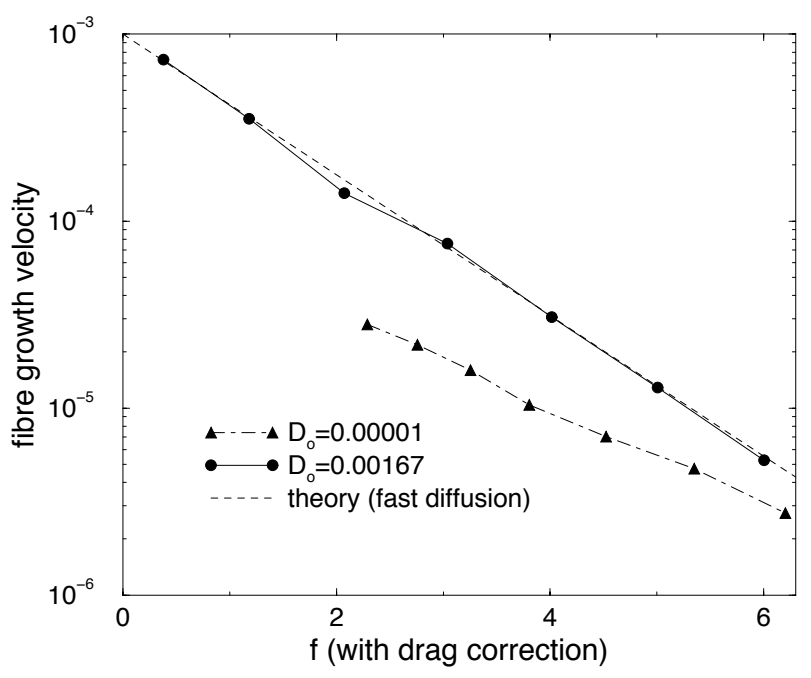

FIG. 4: 
(a)

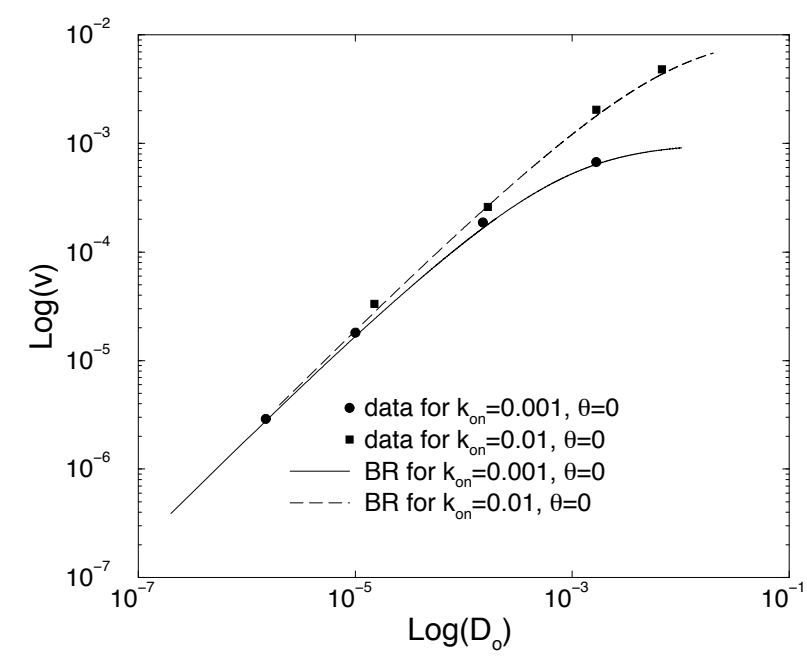

(b)

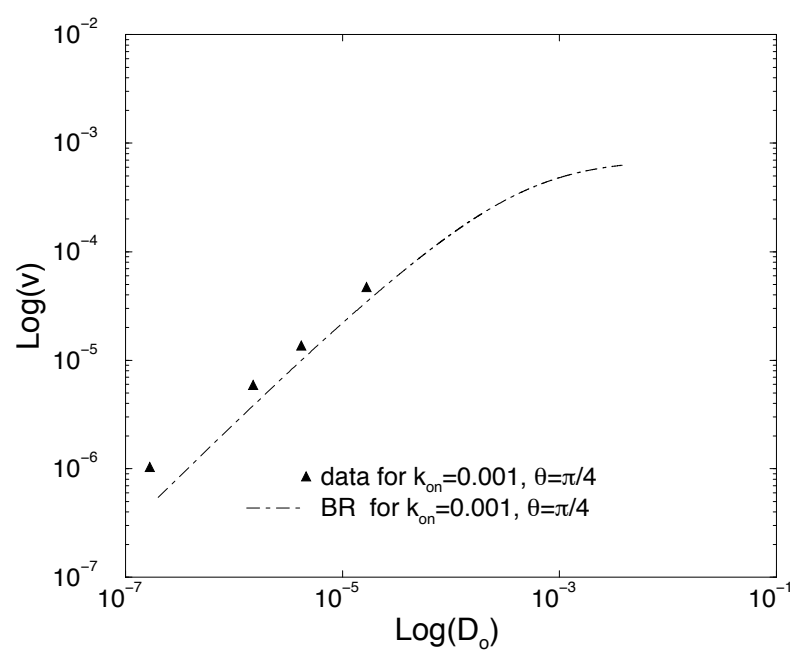

FIG. 5: 

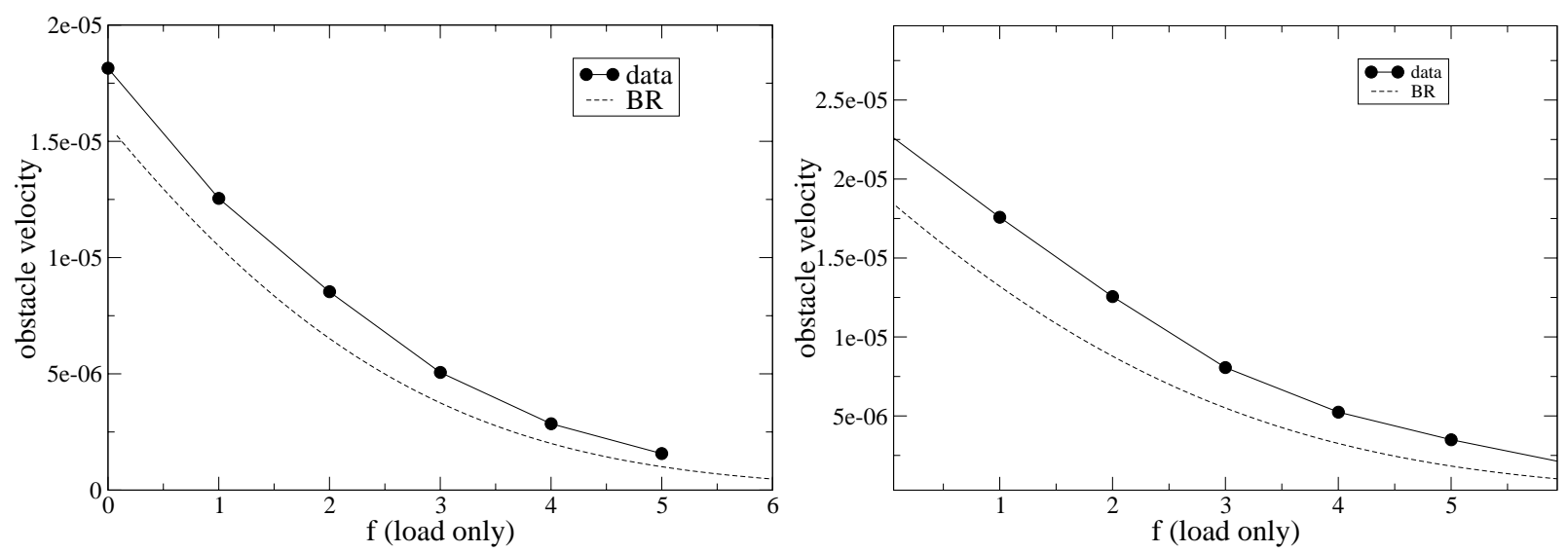

(c)

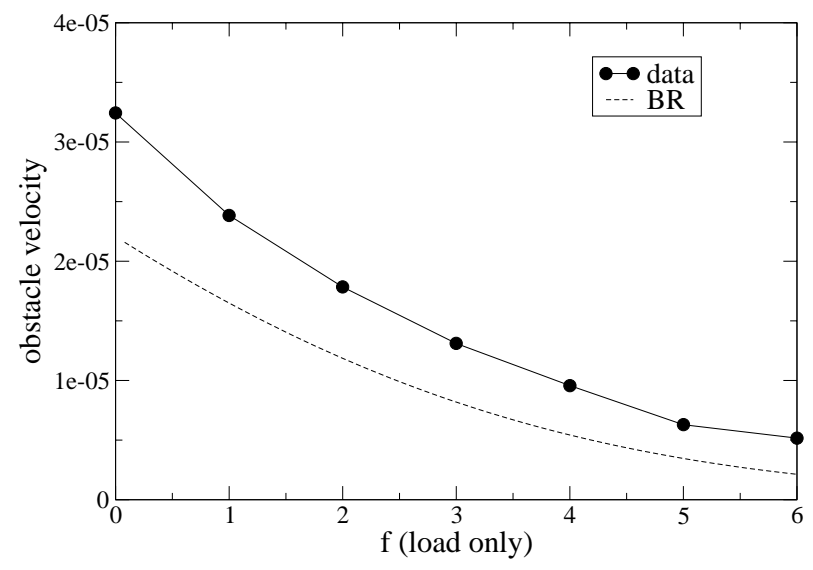

FIG. 6: 
(a)

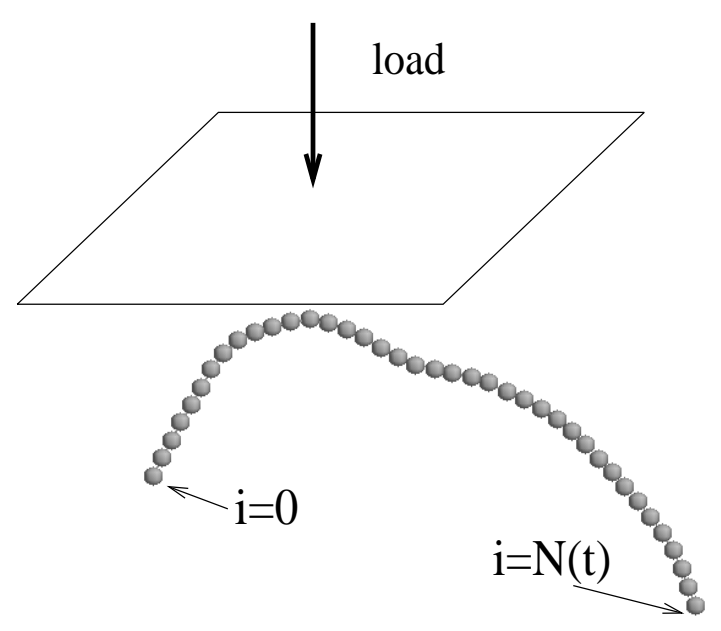

(b)

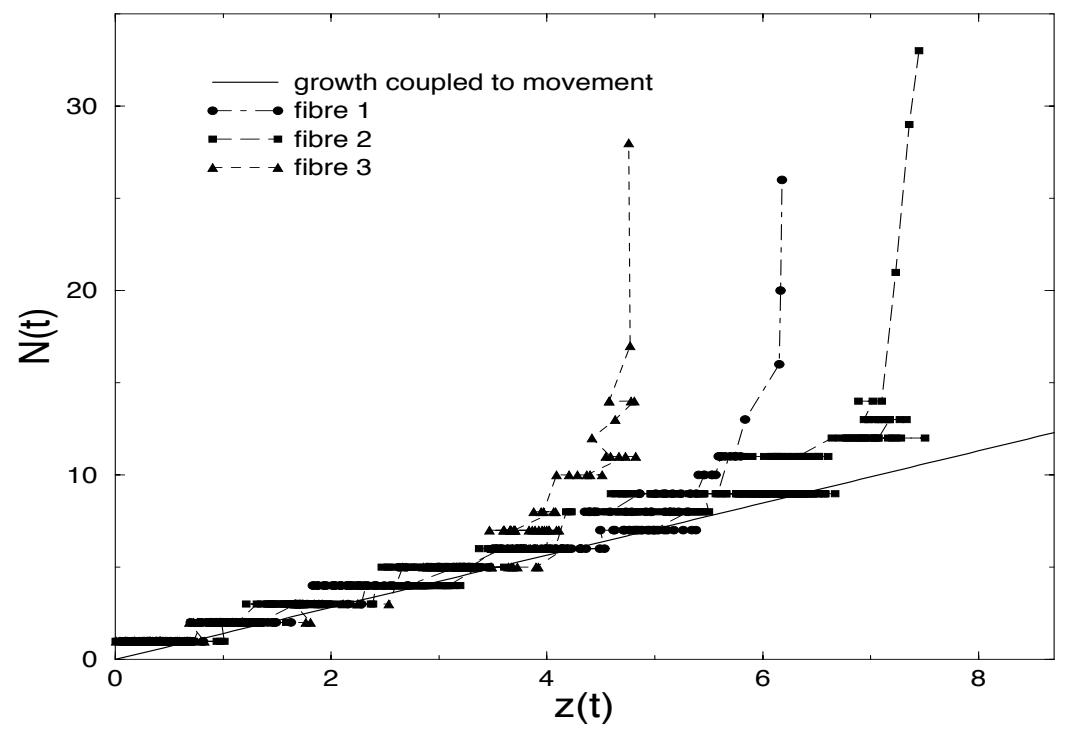

FIG. 7: (a)

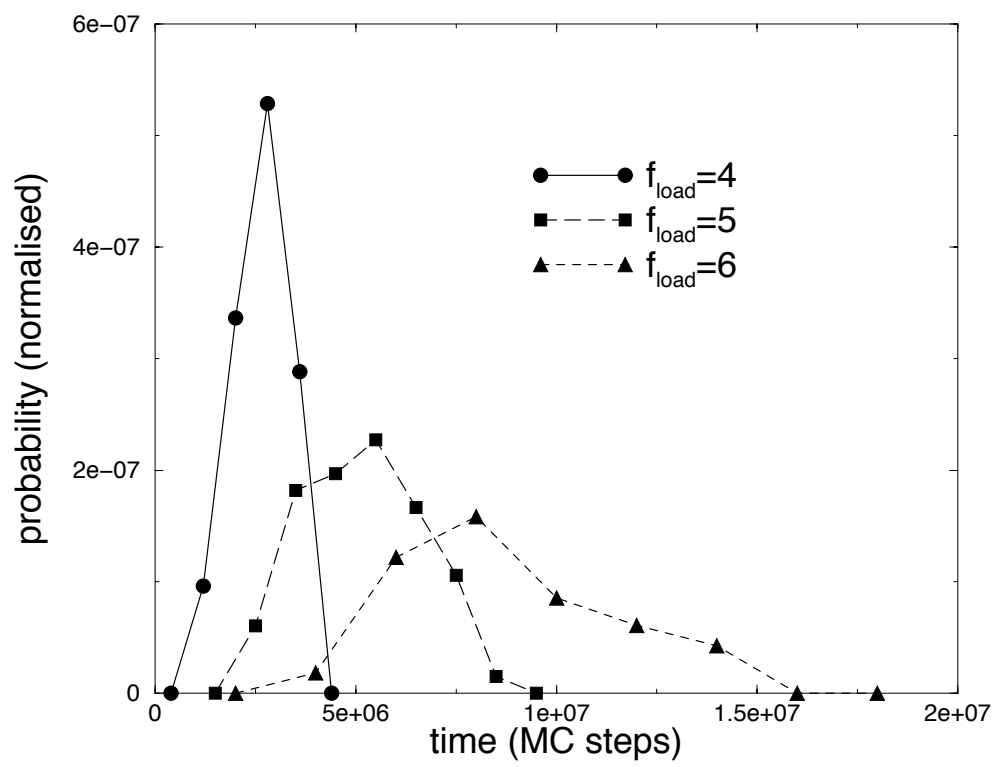

FIG. 8: (b)

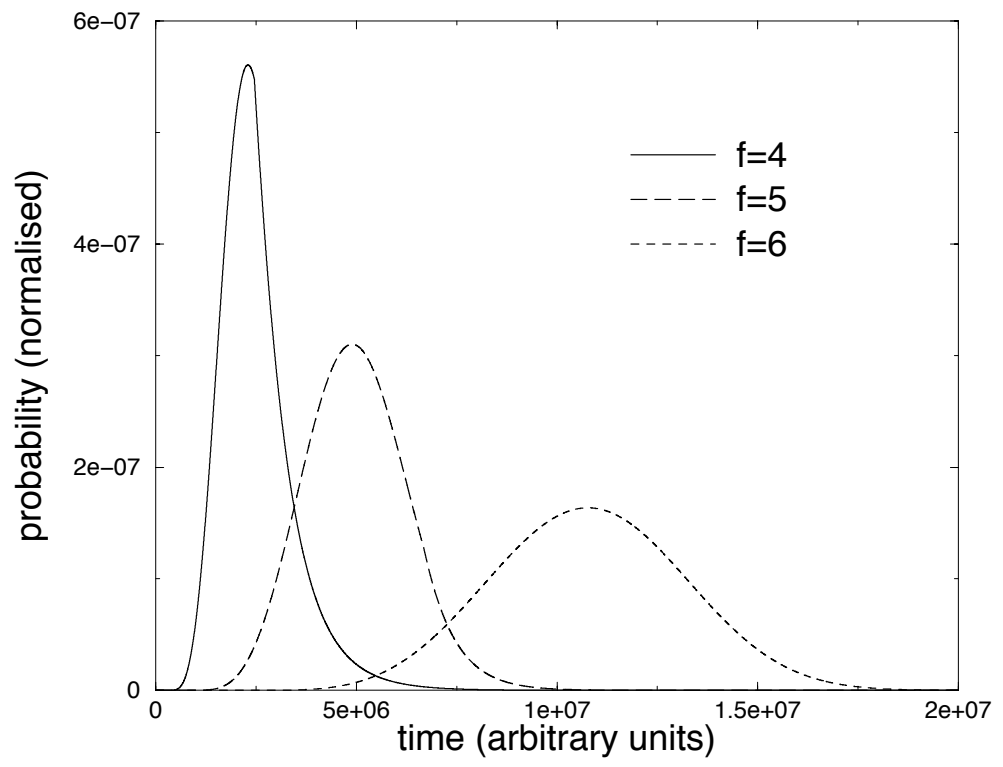


(a)

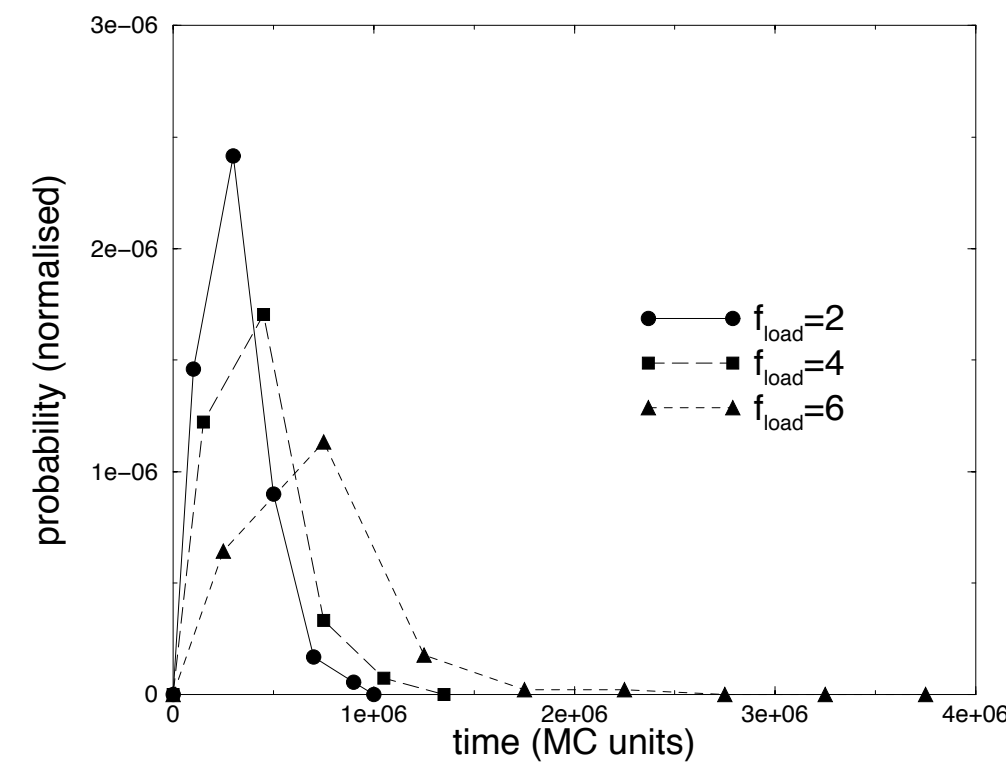

(b)

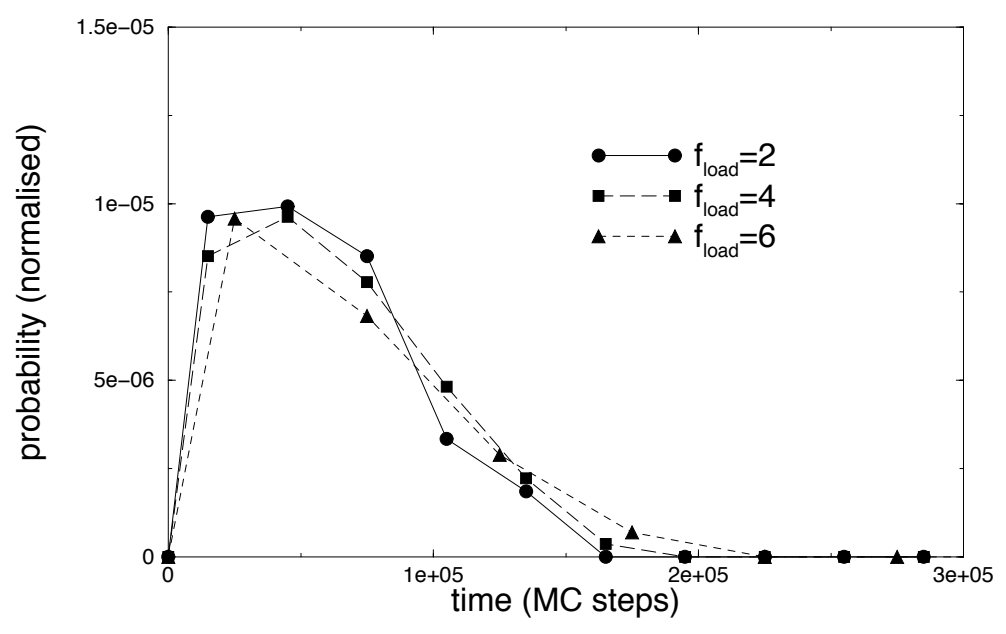

FIG. 9:

(a)

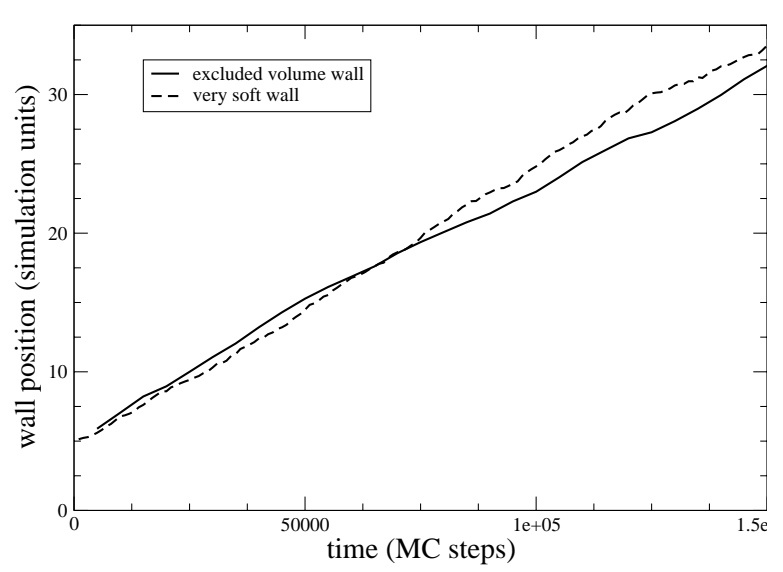

(b)

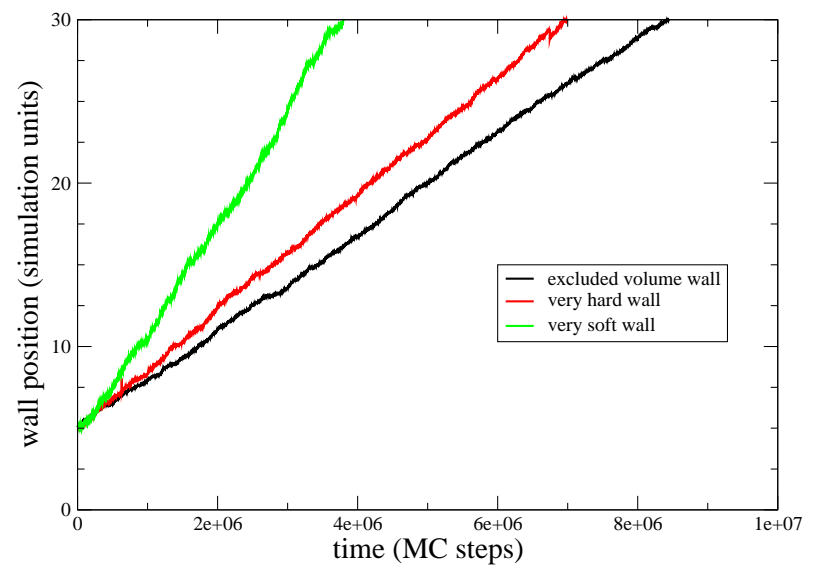

FIG. 10: 


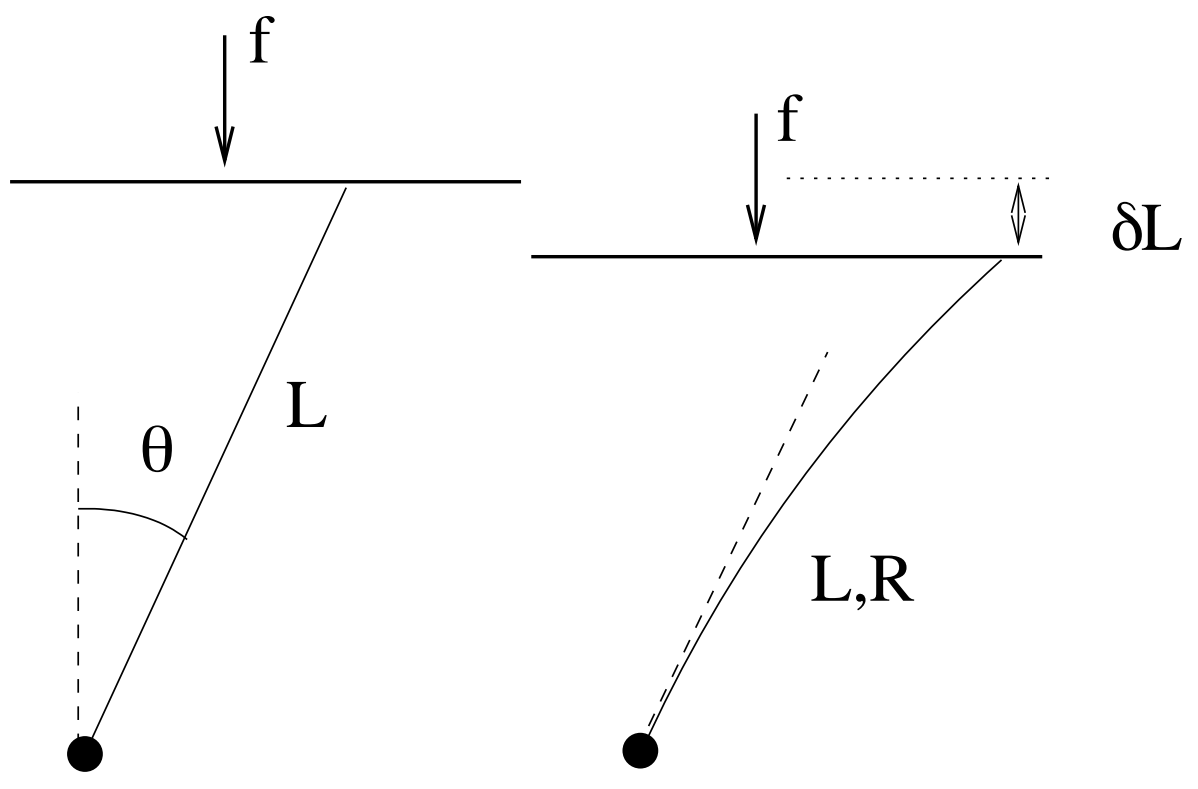

FIG. 11: 